\title{
Catalogue of cataclysmic binaries, low-mass X-ray binaries and related objects (Seventh edition)
}

\author{
H. Ritter ${ }^{1}$ and U. Kolb ${ }^{1,2}$ \\ ${ }^{1}$ Max-Planck-Institut für Astrophysik, Karl-Schwarzschild-Str. 1, 85741 Garching, Germany \\ 2 Department of Physics and Astronomy, The Open University, Milton Keynes MK7 6AA, UK
}

Received 21 January 2003 / Accepted 29 January 2003

\begin{abstract}
The catalogue lists coordinates, apparent magnitudes, orbital parameters, and stellar parameters of the components and other characteristc properties of 472 cataclysmic binaries, 71 low-mass X-ray binaries and 113 related objects with known or suspected orbital periods together with a comprehensive selection of the relevant recent literature. In addition, the catalogue contains a list of references to published finding charts for 635 of the 656 objects, and a cross-reference list of alias object designations. Literature published before 1 January 2003 has, as far as possible, been taken into account. All data can be accessed via the dedicated catalogue webpage at http://www.mpa-garching.mpg.de/RKcat/ and http://physics.open.ac.uk/RKcat/ and at CDS via anonymous ftp to cdsarc.u-strasbg.fr (30.79.128.5) or via http://cdsweb.u-strasbg.fr/cgi-bin/qcat?J/A+A/404/301. We will update the information given on the catalogue webpage regularly, initially every six months.
\end{abstract}

Key words. catalogs - stars: novae, cataclysmic variables - stars: binaries: close

\section{Introduction to the 7th edition}

Five and a half years after the publication of the previous (6th) edition of the Catalogue of Cataclysmic Binaries, Low-Mass X-Ray Binaries and Related Objects (Ritter \& Kolb 1998, hereafter RK98), the amount of new literature and the number of new objects to be included have again grown so much that it seems worthwhile to publish an updated edition. The philosophy and the purpose of this catalogue (now in its 7th edition) have been outlined in the preface to the 3rd edition (Ritter 1984, hereafter R84) and will not be repeated here. Rather we briefly recall some of the developments which, over the past five and a half years, have had (and still have) a major impact on this catalogue:

1. The online and current edition of A Catalog and Atlas of Cataclysmic Variables by Downes et al. (2001, hereafter referred to as DWSRKD) is now the primary source of accurate coordinates and finding charts for cataclysmic variables. The DWSRKD catalogue supersedes earlier editions by Downes \& Shara (1993) and Downes et al. (1997), includes an updated version of A Reference Catalogue and Atlas of Galactic Novae by Duerbeck (1987), and is supplemented by orbital period information by the present authors.

2. An increasing number of CVs are found serendipitously from large surveys such as e.g. the Sloan

Send offprint requests to: $\mathrm{H}$. Ritter,

e-mail: hsr@mpa-garching.mpg.de
Digital Sky Survey (see e.g. Szkody et al. 2002), or from The All Sky Automated Survey at http://www . astrouw . edu.pl/ gp/asas/asas.html.

3. As a result of systematic searches for potential double degenerate Type Ia supernova progenitors (see e.g. Napiwotzki et al. 2001; Marsh 2000; Morales-Rueda et al. 2002) the number of detached short-period double white dwarf systems has increased dramatically and is likely to continue to grow sustantially in the future.

4. We acknowledge the continuing contributions of amateur astronomers to the field of cataclysmic variables, in particular to tracking down the superhump periods of SU UMa stars. Results of such amateur activities can e.g. be found on the webpages of the Variable Star Network (VSNET) at http://vsnet.kusastro.kyoto-u.ac.jp/vsnet/ and of the Center for Backyard Astrophysics (CBA) at http://cba.phys. columbia.edu/.

5. Due to the much increased sensitivity and spatial resolution achievable with present-day X-ray satellites such as Chandra or XMM-Newton, as well as with large groundbased optical telescopes (e.g. the VLT or the Keck telescopes) the number of cataclysmic variables (CVs) and low-mass X-ray binaries (LMXBs) found in globular clusters and in external galaxies (M31, M 51) is also rapidly increasing.

6. Gänsicke \& Kube (2002) are in the process of establishing CVeat, a data base on CVs that aims at a comprehensive collection of all available data on CVs, and to make them 
electronically available to the community. Thus its aims go way beyond those of DWSRKD or the present catalogue.

In view of the activities by Gänsicke \& Kube (2002) and the fact that with DWSRKD there is already an online CV catalogue one might ask whether publishing yet another one is justified. DWSRKD and this catalogue have existed in parallel in paper form before going electronic, and are complementary as far as CVs are concerned. Our catalogue focuses on data related to the objects' binary nature, while DWSRKD gives data that are useful for observational programmes. In addition, our catalogue includes LMXBs and related objects, for which there is no comparable alternative source (the most recent catalogue for LMXBs is by Liu et al. 2001). The CVcat project by Gänsicke \& Kube (2002), on the other hand, is still under development, and it remains to be seen whether this project will render our catalogue superfluous.

Compared with the 6th edition, the number of objects listed has increased by almost $60 \%$. Accordingly the current version of this catalogue provides tabulated data and references for 656 objects (472 cataclysmic binaries, 71 low-mass X-ray binaries and 113 related objects).

No attempt has been made to provide a complete bibliography. Rather, the aim is to give a selection of the most recent and most relevant references that should allow the user to navigate through the literature addressing mainly the binary properties of the objects in question. References that were already included in RK98 are only repeated if they are required for cross-reference (see below). Yet more references can be found in the previous four editions (R84; Ritter 1987, hereafter R87; R90; and RK98) of the catalogue. Accordingly, the 7th edition provides:

- the tables for all three object classes (cataclysmic binaries, low-mass X-ray binaries and related objects) in full;

- for each catalogued object a selection of references to the literature, published after 30 June 1997 (the deadline of RK98). Earlier references are only included if they are needed for cross-reference, or in cases where there have been few or no new publications of relevance;

- a list of selected references to published finding charts. Additional references may be found in previous editions;

- the Who's Who? file, a cross-reference list of alias names of the objects catalogued.

Thus the catalogue is complete and self-contained in the tables and in giving cross-references to alternative object designations.

Every effort has been made to avoid errors and to keep the lists up to date. Nevertheless, the authors are well aware of the fact that also this edition will contain errors and may be incomplete with regard to the criteria stated in the preface to the 3rd edition (R84). It is certainly incomplete with respect to the references quoted.

All the tabular material contained in this catalogue is published in electronic form only. It is available from the dedicated catalogue webpages at the MPA http://www.mpa-garching.mpg.de/RKcat/, and the OU http://physics.open.ac.uk/RKcat/, or in electronic form at the CDS via anonymous ftp to cdsarc.u-strasbg. fr (130.79.128.5) or via http://cdsweb.u-strasbg.fr/ cgi-bin/qcat?J/A+A/404/301.

In addition to the electronic version we provide postscript files for a printable stand alone version at both websites.

For the current version of this catalogue, literature published before 1 January 2003 has, as far as possible, been taken into account.

For the future it is our intention to provide semi-annual updates of the catalogue on the dedicated catalogue webpages.

\section{Description of the catalogue}

The objects listed in this catalogue are subdivided into three main object classes, i.e. into Cataclysmic Binaries, Low-Mass $\mathrm{X}$-Ray Binaries and Related Objects. The defining characteristics of the three object classes used here are the following:

Cataclysmic Binaries are semi-detached binaries consisting of a white dwarf primary (or a white dwarf precursor) and a low-mass secondary which is filling its critical Roche lobe. The secondary is not necessarily unevolved. It may even be a highly evolved star as for example in the case of the AM CVn-type stars. A more detailed description of the main characteristics of these objects may be found in Warner (1995) or Hellier (2001).

In addition, we list among the cataclysmic binaries also the supersoft binary X-ray sources, because these too are semidetached binaries containing a white dwarf, though one in a state of sustained nuclear burning. More information about these objects can be found in Greiner (1996, 2000).

Low-Mass X-Ray Binaries are semi-detached binaries consisting of either a neutron star or a black hole primary, and a low-mass secondary which is filling its critical Roche lobe. Observationally they are distinguished from the luminous, massive X-ray binaries by the following main properties: in general the spectra of the low-mass X-ray binaries (at maximum light) are devoid of normal stellar absorption features. The ratio of their X-ray to optical luminosities is much larger than unity (typically it ranges from $\sim 10^{2}$ to $\sim 10^{4}$ ). A more detailed description of the main characteristics of these objects may be found in the review articles in Lewin et al. (1995).

Related Objects are detached binaries consisting of either a white dwarf or a white dwarf precursor primary and of a low-mass secondary. The secondary may also be a highly evolved star. Further information may be found e.g. in Ritter (1986), Bond (1989), or de Kool \& Ritter (1993). With one possible exception (HD 49798) we do not list among the related objects detached binaries containing a neutron star, or, for the lack of known objects, a black hole. Thus we explicitly exclude binary radio pulsars from our compilation because these are documented elsewhere (e.g. in the Princeton pulsar catalogue, Taylor et al. 1993) which is available online at http://pulsar.princeton. edu/pulsar/catalog. shtml. 
According to the subdivision into these three object classes the catalogue consists of three major parts, hereafter referred to as catalogue sections. Each of the three catalogue sections is further subdivided into a table section, where a few characterizing parameters of the object are tabulated, and a reference section, where a selection of references is given. Within each of the table sections, the objects are listed in order of decreasing orbital period. In the corresponding reference section, however, the objects are listed in lexigraphical order.

Limited information about where the values given in the tables are taken from is provided as follows: at the end of a reference from which a given quantity, say XYZ, was taken, this quantity is given in parenthesis, i.e. as (XYZ). The quantities for which this is done are: the periods (Orb.Per., 2. Per., 3. Per., 4. Per.), the spectral types (Spectr1, Spectr2), the mass ratio (M1/M2), the orbital inclination (Incl), the masses (M1, M2), and, where appropriate, the radii (R1, R2) and the eccentricity (e).

The catalogue is supplemented by a list giving references to published finding charts of the objects. In this separate section, the objects of all three classes are merged and listed in lexigraphical order. The full form of abbreviated references used is given at the end of this section.

Finally, the Who's Who? file contains a cross-referenced list of alias names of the objects catalogued. In order to keep this list short, the full list of alternative object names appears only once for each object and is to be found under the standard name used in this catalogue. If an object is sought under one of its alternative names, reference to the standard name is given. Wherever possible the variable name given in the 4th edition of the General Catalogue of Variable Stars (Kholopov et al. 1985a, 1985b, 1987), or in its online version at http: //www.sai.msu.su/groups/cluster/gcvs/gcvs/, or in the Name Lists of Variable Stars (up to and including the 76th list, Kazarovets et al. 2001) is used as the standard name here. This section includes also a list of references to various catalogue acronyms that appear in this compilation. More complete information of this kind may be found in The First Dictionary of the Nomenclature of Celestial Objects by Fernandez et al. (1983), its supplements Lortet (1986a, 1989b), and Lortet \& Spite (1986), in the Second Reference Dictionary of the Nomenclature of Celestial Objects by Lortet et al. (1994), or online via the Centre de données astronomiques de Strasbourg $(C D S)$ at http://vizier.u-strasbg.fr/cgi-bin/Dic, or from the Astronomical Data Center (ADC) at http://adc.gsfc.nasa.gov/adc/.

Acknowledgements. We wish to thank H.-C. Thomas and V. Burwitz for keeping us informed about the latest results regarding the optical identification and follow-up observations of new CVs from the ROSAT All Sky Survey. We also thank J. Thorstensen for providing us with information on numerous new CVs with newly measured periods prior to publication. This research has made use of the SIMBAD database, operated at CDS, Strasbourg, France.

\section{References}

Bond, H. E. 1989, in Planetary Nebulae, ed. S. Torres-Peimbert (Dordrecht: Kluwer), 251

de Kool, M., \& Ritter, H. 1993, A\&A, 267, 397

Downes, R. A., \& Shara, M. M. 1993, PASP, 105, 127

Downes, R. A., Webbink, R. F., \& Shara, M. M. 1997, PASP, 109, 345

Downes, R. A., Webbink, R. F., Shara, M. M., et al. 2001, PASP, 113, 764 (DWSRKD); tabular material available only online at http://icarus.stsci.edu/ downes/cvcat/index.html

Duerbeck, H. W. 1987, Space Sci. Rev., 45 (1/2)

Fernandez, A., Lortet, M.-C., \& Spite, F. 1983, A\&AS, 52 (4)

Gänsicke, B. T., \& Kube, J. 2002, at http://astrocat.uni-goettingen.de/

Greiner, J. (ed.) 1996, Supersoft X-Ray Sources, Lecture Notes in Physics Vol. 472 (Berlin: Springer-Verlag)

Greiner, J. 2000, at http://www.aip.de/People/JGreiner/ sss/ssscat.html

Hellier, C. 2001, Cataclysmic Variable Stars (Berlin: Springer-Verlag)

Kazarovets, E. V., Samus, N. N., \& Durlevich, O. V. 2001, IBVS No. 5135

Kholopov, P. N., Samus, N. N., Frolov, M. S., Goranskij, V. P., et al. 1985a, General Catalogue of Variable Stars, 4th edition (Moscow: Nauka), vol. I

Kholopov, P. N., Samus, N. N., Frolov, M. S., et al. 1985b, General Catalogue of Variable Stars, 4th edition (Moscow: Nauka), vol. II

Kholopov, P. N., Samus, N. N., Frolov, M. S., Goranskij, V. P., et al. 1987, General Catalogue of Variable Stars, 4th edition (Moscow: Nauka), vol. III

Lewin, W. H. G., van Paradijs, J., \& van den Heuvel, E. P. J. (ed.) 1995, X-Ray Binaries, Cambridge Astrophysics Series vol. 26 (Cambridge: Cambridge Univ. Press)

Liu, Q. Z., van Paradijs, J., \& van den Heuvel, E. P. J. 2001, A\&A, 368,1021

Lortet, M.-C. 1986a, A\&AS, 64, 303

Lortet, M.-C. 1986b, A\&AS, 64, 325

Lortet, M.-C., \& Spite, F. 1986, A\&AS, 64, 329

Lortet, M.-C., Borde, S., \& Ochsenbein, F. 1994, A\&AS, 107, 193

Marsh, T. R. 2000, New Astron. Rev., 44, 119

Morales-Rueda, L., Maxted, P. F. L., Marsh, T. R., North, R. C., \& Heber, U. 2003, MNRAS, 338, 752

Napiwotzki, R., Christlieb, N., Drechsel, H., et al. 2001, AN, 322, 411

Ritter, H. 1984, A\&AS, 57, 385 (3rd edition, R84)

Ritter, H. 1986, A\&A, 169, 139

Ritter, H. 1987, A\&AS, 70, 335 (4th edition, R87)

Ritter, H. 1990, A\&AS, 85, 1179 (5th edition, R90)

Ritter, H., \& Kolb, U. 1998, A\&AS, 129, 83 (6th edition, RK98)

Szkody, P., Anderson, S. F., Agüeros, M., et al. 2002, AJ, 123, 430

Taylor, J. H., Manchester, R. N., \& Lyne, A. G. 1993, ApJS, 88, 529

Warner, B. 1995, Cataclysmic Variable Stars, Cambridge Astrophysics Series vol. 28 (Cambridge: Cambridge Univ. Press) 